\title{
Respiration chamber calorimetry and doubly labeled water: two complementary aspects of energy expenditure?
}

\author{
Yves Schutz ${ }^{1}$
}

\section{Introduction}

Energy is a physical abstraction, which cannot be measured as such, but which is easily transformed from one form to another form. As a result when physiologists speak about "energy expenditure" they really mean, "heat production", i.e., energy released as heat, as an end-product of the biochemical endogenous reactions (and useful «work») assessed by indirect calorimetry. Since humans are homeotherm, the almost constancy of internal body temperature requires that the total heat produced by the body must be quickly eliminated from the body via different processes of heat dissipation. This is classically called total "heat losses" that is assessed by direct calorimetry, which constitutes the sum of radiative, evaporative (via the skin and breath), convective and conductive routes [1].

Note that temporal synchronization between the rate of heat loss and heat production is not instantaneous, due to different thresholds and lag time in the onset of these physiological processes of regulation and in difference in calorimetry response time, so that heat loss may lag behind the heat production. This temporal gap in heat imbalance explains the well-known phenomenon described as «circadian rythm» of central body temperature higher core temperature during diurnal activities, after eating a meal and after intense exercise (due to net heat storage) and lower central temperature at night during sleep (due to net heat released).

In brief, the two techniques to be discussed (respiration chamber and D2O18) estimate heat production (total energy expenditure, TEE) either by indirect calorimetry, i.e., by the measurement of both oxygen consumption (VO2) and $\mathrm{CO} 2$

Yves Schutz

Yves.Schutz@unifr.ch

1 Department of Integrative Physiology, Faculty of Medicine, University of Fribourg, Fribourg, Switzerland production $(\mathrm{VCO} 2)$ in an open-system respiration chamber or «doubly indirectly» using D2O18, which provides a single estimate of $\mathrm{CO} 2$ production over 1 week or 2 .

For a nutritionist, the importance of assessing TEE in free-living conditions is immense in order to obtain information on total energy requirement of individuals as function of exogenous and endogenous factors, since energy intake assessment by conventional methods is usually unreliable.

For an ecologically oriented and provocative-minded researcher, the data generated by D2O18 may appear to be an expensive way to measure human greenhouse gas emission ( $\mathrm{CO} 2$ ), an information totally forgotten in the issue of contribution of humans $\mathrm{CO} 2$ production to global warming on earth.

The evaluation of two distinct methodologies in human research is not as straightforward as it looks, since it is never a «black or white» judgment. It ought to be objectively based on data publications in peer-reviewed papers and not perturbed by a biased judgment or conflict of interest.

Yet an investigator working for decades on one of these methods, who has participated in its early development, and who has published a myriad of scientific articles and review papers on the method, is very knowledgeable and he/she will progressively become a leader in the field. Logically he (she) will not take the risk of "shooting a bullet in his own feet" by particularly highlighting the shortcomings of his (her) technique of choice. As a result, the definitive judgment of a methodology used for research constitutes a risky and sensitive task, since prejudice could exist and the technique is in constant evolution so that it becomes more accurate and precise over decades with the development of new technology and innovation.

The authorities who have spent their life on one of these two methods have made the domain grow and have a legitimate tendency to promote it by an appropriate communication to the research world, the final aim being to generate more data on TEE in the world. However, one 
should keep in mind that, besides the relative attractiveness and value of each method, these methods have a number of technical and biological limitations [1,2] some of the most important being outlined below.

\section{D2018}

The D2O18 method was conceptualized by the genius Lifson in the middle of the XXth century. It was first applied to zoology by measuring small animals energy expenditure. Then, from the middle of 1980s decade, experimental studies using D2O18 in humans were published and validated against the respiration chamber [3]. Briefly, $\mathrm{CO} 2$ production rate $(\mathrm{VCO} 2)$ is calculated from the total body water pool based on $\mathrm{O} 18$ and D2 dilution spaces multiplied by the difference in the fractional turnover rates of $\mathrm{O} 18$ and $\mathrm{D} 2$, corrected from the rate of water loss occurring via routes subjected to isotopic fractionation. The principle is that D2 is eliminated from the body as water (urinary excretion, insensible water loss, and sweat), while ${ }^{18} \mathrm{O}$ leaves the body both as water $\mathrm{H}_{2} \mathrm{O}$ and $\mathrm{CO}_{2}$. Therefore, the rate of $\mathrm{CO}_{2}$ production ( $\mathrm{VCO} 2$ ) can be calculated by difference between the elimination rate (turnover) of the two pools D2 and O18.

Clear mathematical equations allow to calculate TEE by D2O18. Note that the total number of variables in the different mathematical equations to arrive at $\mathrm{VCO} 2$ production is 9 plus 2 constants, from which the equation for isotope dilution spaces calculation includes 5 variables and 1 constant. Using indirect calorimetry respiration chamber, VCO2 calculation is more straightforward and requires only 4 variables and 1 constant. The conversion of VCO2 to TEE needs only 1 variable (energy equivalent of $1 \mathrm{~L} \mathrm{CO}_{2}$ ), which depends upon the respiration quotient (RQ).

Speakman [4], after 25 years of utilization of D2O18 in research, pointed out that "validation studies show that for groups of subjects the method works well. However, the precision is still relatively poor (8-9\%). Consequently the method is not yet sufficiently refined to provide estimates of individual energy expenditures." Is it still true today?

Subsequent refinements in the equations used (for example, tailored factors and constant in the model adapted to extreme environmental conditions) were indeed made, but «one cannot transformed a Volkswagen Rabbit into a Ferrari... if you do not change the engine»!

\section{Respiration chamber}

Respiration chambers are considered as classic method, ingeniously implemented more than one century ago by Max von Pettenkofer in Germany and Atwater in USA [5]. Today, there are many functional chambers dispersed around the world, either built by engineers (home-made built) or inspired from other existing metabolic chambers that showed good accuracy and precision [6]. Semi-portable and rugged respiration chambers have also been built for nutritional and metabolic research utilization in 3rd world countries where harsh conditions occurs. Indeed a transportable (ambulatory) respiration chamber was built more than 20 years ago for use in West Africa [7], following a collaborative project between the University of Lausanne (Department of Physiology) and the University of Cambridge (UK).

Since a decade ago, commercial «ready to use» respiration chambers are also on the market. Although the total cost of the system appears to be elevated, the running cost is expected to be low, in particular for «home made» systems of top quality.

The human subjects, fully confined in the system, must adapt to the particular conditions since there is no social interaction during the measurements, disregarding the visual and audio contact with one of the investigators.

Typically, the subjects spend a minimum of $24 \mathrm{~h}$ continuously, in a cross-sectional design, and up to a week (or more) in nutritional intervention studies, for example, in the first short-term overfeeding study performed 35 years ago [8].

For the investigator, the limitation of restrained space of the room is largely offset by the standardized environmental conditions (pre-selected by the investigators) and the full control of food intake (adjusted for size) and therefore nutritional tailored to each subject in order to target energy equilibrium. This allows identical research conditions for the entire subjects studied.

Generally the subject quickly adapts to the system. In some occasions, dummy measurements are made for a few hours prior to the real study.

\section{Respiration chamber used as a reference method or "golden standard"}

\section{Validation of respiration chambers vs direct calorimetry}

Before being used as a reference value for evaluating D2O18, the respiration chamber should also be validated itself against another independent method. Indirect calorimetry measurements have been compared to concomitant measurements of direct calorimetry using hybrid system [9].

In small size hybrid calorimeters, the rate of total heat losses and total heat production, after a short transient adaptive phase, were essentially the same under steady-state conditions [10].

In longer-term comparison in respiration chambers, the results corroborate that direct and indirect calorimetry give 


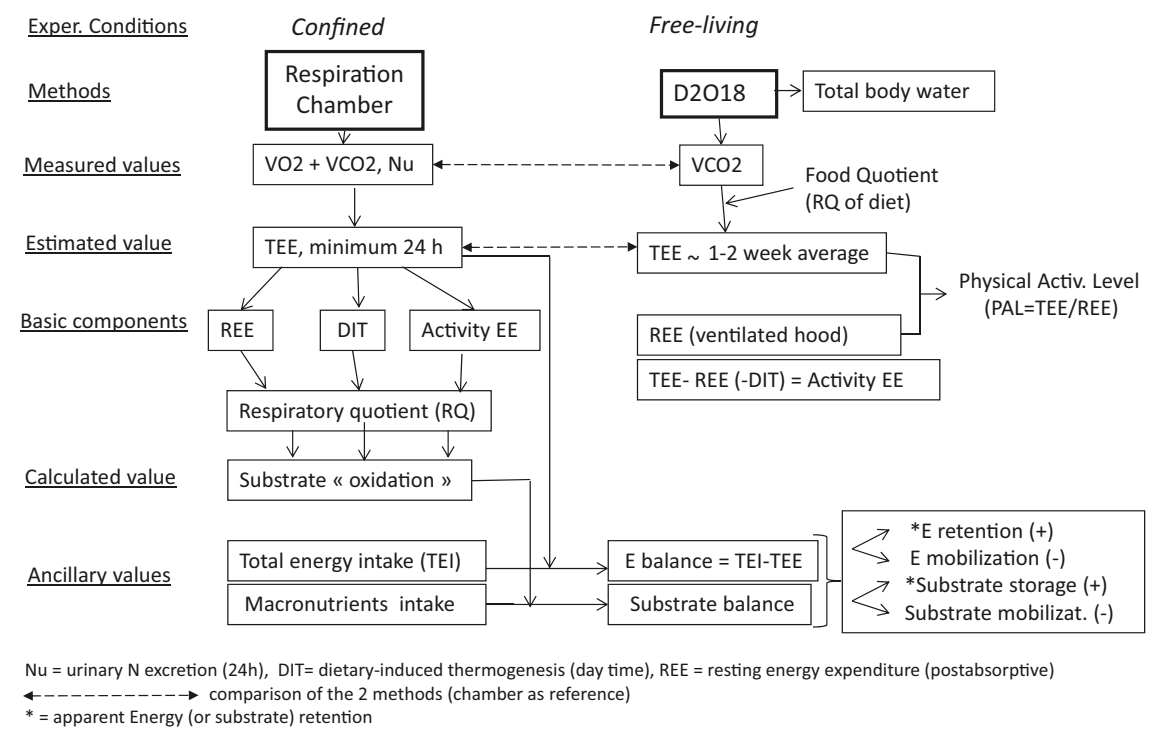

Fig. 1 Overview of comparative features between the two methodologies for assessing total energy expenditure (TEE): it clearly shows that the amount of information obtained in a whole-body indirect calorimetric chamber over $24 \mathrm{~h}$ far outweigh the single (1 week average) TEE value obtained with D2O18. A short list of advantages and shortcomings is summarized briefly. The strong point of D2O18 is: inconspicuous and non-invasive free-living evaluation of TEE with no constraint or restriction for the subject. The strong points of the

almost identical values (with $99.9 \%$ accuracy for both systems) for total heat production and total heat losses when integrated over a $24-\mathrm{h}$ period and when the initial and final chamber conditions were unchanged [11].

Whole-body indirect calorimetry chambers have been used as a reference method to «validate» D2O18 methods by comparing 1 week average $\mathrm{CO}_{2}$ production (respectively TEE) measurements by D2O18 vs that measured by the respiration chamber.

The results in terms of accuracy were judged as good when the average of a group of subjects is considered (much below 5\% inaccuracy) but in a given subject the range in $\%$ error was large ( -7 to $22 \%$ ) in the early study [3] but it had the same CV of relative error (9\%) in two different experimental studies 20 years a part [12].

A comparison scheme of the variables measured and amount of information obtained for each system is outlined in Fig. 1. In free-living situation, due to the large potential error of measuring total energy intake combined to some uncertainty of assessing TEE by D2O18 at the individual level, the perspective of accurately measuring the magnitude of daily or weekly energy imbalance (i.e., energy gap) in a given individual remains, hélas, elusive.

\section{Criteria to select a system}

The main criteria is obviously free-living vs confined conditions but several additional factors can be mentioned such respiration chamber are: (1) profile of EE and substrate oxidation assessed over $24 \mathrm{~h}$ (or more), in standardized environmental and nutritional conditions. (2) high precision and accuracy of the system over $24 \mathrm{~h}$. The main weak points of D2O18 are: (1) insufficient accuracy of TEE at the individual level. (2) Provides one single integrated average value of TEE over 1-2 weeks, at a high cost. The weak point of the respiration chamber is: confined conditions in a room with little social interactions

as, the objectives of the research, the degree of non-invasiveness, the duration of the study, the technical training required when using the methods (D2O18), the degree of portability of the system for utilization in field studies, the technical reliability, the budget available and last but not least, the relative error (accuracy and precision) that can be tolerated for the experimental studies.

\section{D2018 and respiration chamber are the two sides of the same coin}

We should acknowledge that each system can benefit from the other, in particular D2O18 from the respiration chamber for the following reasons:

1. D2O18 needs an accurate and precise respiration chamber to validate its performance over several days.

2. D2O18 also requires a classical bedside indirect calorimeter (for example, using a ventilated hood system) for assessing resting EE in order to calculate the «Physical Activity Level» index (PAL = Total EE/resting EE), a key information often reported in parallel to TEE.

3. D2O18 also needs quantitative food intake information (for the calculation of food quotient), in order to correctly convert $\mathrm{VCO} 2$ into $\mathrm{EE}$, using the energy equivalent of $\mathrm{VCO} 2$. The latter varies widely 
depending upon the respiratory quotient assumed to be equal to the food quotient.

In corollary, respiration chamber can benefit from D2O18 method to measure the subject under free-living conditions and analyze the TEE of a subject inside (spontaneous physical activity) vs outside the chamber [13, 14]. The degree of correlation in TEE in the two measurement situations in healthy subjects have been judged good to excellent. As expected, TEE in free-living conditions was almost invariably higher than in the confined conditions. Furthermore, clinical studies in metabolic chambers can also be performed, for example in chronic patients (such as COPD) to evaluate whether the different components of TEE under controlled conditions are abnormally affected [15]. In contrast, in patients with acute illness, the method of choice remains bedside validated indirect calorimetry.

In summary, we can admit that the respiration chamber calorimetry and doubly labeled water constitutes two complementary (non-competitive) aspects of energy expenditure. However, the recent evolution of these two techniques has been modest: the «skeleton» (basic principle and basic mathematical equations) of both respiratory chamber and D2O18 have remained essentially unchanged. However, refinement can still be made in terms of quicker response time and stabilization of RQ volatility in the chamber (when short calculation periods are considered such as min-by-min). For D2O18, lately, a cheaper analytical process of $\mathrm{D} 2 \mathrm{O} 18$ has been developed using of laserbased spectroscopy rather than a mass spectrometry.

Finally, the 50th anniversary of European Journal of Clinical Nutrition is a good opportunity to recall the wide audience for the clinical and non-clinical nutrition domain, in which the energy expenditure studies have played an important role.

I must confess my contentment to have been able to publish in European Journal of Clinical Nutrition, more than 20 years ago (J. Garrow was the editor), the first paper on the potential utilization of satellites based global positioning system (GPS) for free-living measurements of outdoor physical activities, in terms of velocity of displacement and geo-positioning in humans on earth [16].

Acknowledgements The author dedicates this paper to Pr. Hiro Tanaka (Univ. of Fukuoka, Japan) who passed away on April 23, 2018. He leaves behind a whole school of dedicated scientists, to whom he has transmitted his passion for research and his intellectual robustness.

\section{Compliance with ethical standards}

Conflict of interest The authors declare that they have no conflict of interest.

\section{References}

1. Jéquier E, Acheson K, Schutz Y. Assessment of energy expenditure and fuel utilization in man. Annu Rev Nutr. 1987;7:187-208.

2. Dulloo AG, Jacquet J, Montani JP, Schutz Y. Adaptive thermogenesis in human body weight regulation: more of a concept than a measurable entity? Obes Rev. 2012;13(Suppl 2):105-21.

3. Schoeller DA, Ravussin E, Schutz Y, Acheson KJ, Baertschi P, Jéquier E. Energy expenditure by doubly labeled water: validation in humans and proposed calculation. Am J Physiol. 1986;250: R823-R830.

4. Speakman JR. The history and theory of the doubly labeled water technique. Am J Clin Nutr. 1998;68:932S-938S.

5. Schoffelen PFM, Plasqui G. Classical experiments in whole-body metabolism: open-circuit respirometry-diluted flow chamber, hood, or facemask systems. Eur J Appl Physiol. 2018;118:33-49.

6. Jéquier E, Schutz Y. Long-term measurements of energy expenditure in humans using a respiration chamber. Am J Clin Nutr. 1983;38:989-98.

7. Charbonnier A, Jones CD, Schutz Y, Murgatroyd PR, Whitehead $\mathrm{RG}$, Jéquier $\mathrm{E}$. A whole body transportable indirect calorimeter for human use in the tropics. Eur J Clin Nutr. 1990;44:725-31.

8. Schutz Y, Acheson KJ, Jequier E. Twenty-four-hour energy expenditure and thermogenesis: response to progressive carbohydrate overfeeding in man. Int J Obes. 1985;9(Suppl 2):111-4.

9. Dauncey J, Murgatroyd PR, Cole TJ. A human calorimeter for the direct and indirect measurement of $24 \mathrm{~h}$ energy expenditure. BJN. 1978;39:557-66.

10. Schutz Y, Jéquier E. Energy needs: assessment and requirement. In: Shils ME, Olson JA, Shike M, editors. Modern nutrition in health and disease, vol. I. 8th ed., Lea \& Febiger; Philadelphia. 1993; pp. 101-11.

11. Rumpler JL, Seale WV. Synchronous direct gradient layer and indirect room calorimetry. J Appl Physiol. 1997;83:1775-81.

12. de Jonge L, DeLany JP, Nguyen T, Howard J, Hadley EC, Redman LM, Ravussin E. Validation study of energy expenditure and intake during calorie restriction using doubly labeled water and changes in body composition. Am J Clin Nutr. 2007;85:73-79.

13. Schutz Y, Jéquier E. Energy expenditure. Lancet. 1986;327:101-2.

14. Kumahara H, Tanaka H, Schutz Y. Physical activity under confinement and free-living conditions. Physiol Behav. 2010;100: $350-6$.

15. Hugli O, Schutz Y, Fitting JW. The daily energy expenditure in stable chronic obstructive pulmonary disease. Am J Respir Crit Care Med. 1996;153:294-300.

16. Schutz Y, Chambaz A. Could a satellite-based navigation system (GPS) be used to assess the physical activity of individuals on earth? Eur J Clin Nutr. 1997;51:338-9. 